\title{
Chapter 23 \\ New Drugs for Recurrent or Metastatic Nasopharyngeal Cancer
}

\author{
Olubukola Ayodele and Lillian L. Siu
}

\section{Introduction}

Nasopharyngeal cancer (NPC) is a rare subset of head and neck cancers with geographical diversity, whereby the incidence is approximately 20-30 cases per 100,000 in Eastern versus 1 per 100,000 in Western countries. Early stage and localized NPC generally carry a good prognosis; however systemic dissemination develops in approximately $20 \%$ of patients with locoregionally advanced disease $[1,2]$. Additionally, about $15 \%$ of patients present with distant metastases at primary diagnosis [3]. For these patients with recurrent or metastatic (RM) NPC, treatment options are largely limited to palliative systemic therapies leading to poor outcomes. Although NPC is a chemosensitive disease with some studies reporting response rates of over $80 \%$ with platinum-based chemotherapy regimens in the first-line recurrent or metastatic setting $[4,5]$, resistance invariably develops; therefore better treatment options are needed. Platinum-containing doublet chemotherapy is generally regarded as the standard first-line treatment for patients with RMNPC. The randomized phase III trial of gemcitabine and cisplatin (GC) versus 5-fluorouracil and cisplatin (PF) in RMNPC established GC as the standard of care first-line regimen. The overall response rate (ORR) in the $\mathrm{GC}$ arm was $64 \%$ compared to the $\mathrm{PF}$ arm of $42 \%$ [6]. Despite the superior outcome of the GC arm that reported a median survival of 29.1 months, RMNPC patients ultimately succumb to their advanced malignancy.

O. Ayodele

Princess Margaret Cancer Centre, University Health Network, University of Toronto,

Toronto, Canada

e-mail: Olubukola.ayodele@uhn.ca

L. L. Siu $(\bowtie)$

Princess Margaret Cancer Centre, Toronto, ON, Canada

e-mail: lillian.siu@uhn.ca 
The pathogenesis of NPC involves genetic, lifestyle, and viral infection factors $[7,8]$. NPC is an Epstein-Barr virus (EBV)-associated cancer in which programmed cell death ligand 1 (PD-L1) expression is upregulated upon EBV activation. This feature is predominately among the WHO class II and III subtypes [9]. Expression of viral proteins in NPC cells can elicit a virus-specific immune response in patients with NPC [9]. Latent membrane protein 1 (LMP-1) expression and interferongamma activation can synergistically induce PD-L1 in NPC cells [10]. In fact, PD-L1 expression is reported to occur in $89 \%$ to $95 \%$ of NPC tumors [10-12]. This increased PD-L1 expression may be associated with better efficacy to immune checkpoint blockade using anti-PD1/L1 antibodies.

Development of new systemic therapies for RMNPC has stagnated in the past 20 years despite the conduct of numerous clinical trials. The exploration of molecular targeted therapy has been hindered by the absence of a definite genetic driver or actionable alterations in this malignancy. There are currently no approved targeted agents for RMNPC and no standard treatment options beyond the first-line setting for patients with platinum-refractory RMNPC.

In this review, we highlight potential new therapeutic options such as immunotherapy and molecularly targeted agents in patients with RMNPC.

\section{Immuno-Oncology in RMNPC}

Histologically, NPC is characterized by heavy infiltration of immune cells within its stroma. These cells consist mostly of CD3+, CD8+, regulatory T cells (Treg), natural killer cells, neutrophils, dendritic cells and mast cells [13]. The co-presence of active cancer cells together with abundant immune infiltrates reflects the underlying functional immune suppression within the NPC tumor microenvironment [14]. These dense immune infiltrates were postulated to be involved in the growth and invasive properties of NPC [15]. Studies have demonstrated an inverse relationship between survival and the density of tumor infiltrating CD8+, neutrophils and mast cells [13]. The immune system plays a critical role in the surveillance, prevention and development of cancer. Evasion of the immune system has been established as a hallmark of cancer [16]. It is therefore highly attractive to manipulate the immune system in such a way as to induce an antitumor response. The various immunotherapeutic strategies that have been employed in the management of RMNPC include immune checkpoint inhibitors, adoptive cell therapies, EBV directed vaccines, personalized cancer vaccines and oncolytic viruses.

\section{Immune Checkpoint Inhibitors (ICI)}

Due to the overexpression of PD-L1 in RMNPC, several single arm phase I/II studies of anti-PD1 antibodies have been conducted in this malignancy. KEYNOTE-028, 
a phase Ib non randomized clinical trial of pembrolizumab (humanized IgG4) was the first single arm study to assess a PD-1 inhibitor in PD-L1 positive RMNPC. The NPC cohort enrolled 27 patients with a mixed background of treatment-naïve or pretreated squamous and non-squamous NPC. The PD-L1 status had to be positive ( $\geq 1$ combined positive score [CPS] using $22 \mathrm{C} 3$ antibody) for trial enrollment. Partial response and stable disease were observed in seven and 14 patients, respectively, for an ORR of $25.9 \%$ and a 1-year overall survival (OS) of 63\% [17]. A similar study by Ma et al. (NCI-9742) investigated the use of another PD-1 inhibitor nivolumab (fully human IgG4) and demonstrated an ORR of 20.5\% and a 1-year OS of 59\%. This was a phase II clinical trial of patients who had progressed after firstline chemotherapy containing platinum with no PD-L1 cut off point included (Table 23.1). A subgroup analysis showed response was higher among patients with PD-L1 positive tumors, defined as $\geq 1$ membrane staining in tumor and immune cells using 22C3 antibody [18] (33\% vs. 13\% for PD-L1 positive versus negative patients respectively).

In another single arm study, Fang et al. [19] reported the findings of a newer PD-1 inhibitor camrelizumab (humanized IgG4) among 93 Chinese patients with RMNPC. This phase I clinical trial had no PD-L1 cut off and demonstrated an ORR of $34 \%$ and 1-year progression free survival (PFS) of $27.1 \%$. In the same report, a separate small cohort of 23 patients who received a combination of GC-based chemotherapy with camrelizumab was included. This combination produced a staggering ORR of 91\%, 6-month PFS and 12-month PFS of $86 \%$ and 61\%, respectively. Other PD-1 inhibitors investigated in single arm phase I/II trials in China include toripalimab (JS001) and tislelizumab (BGB-A317). Their results were presented at the American Society of Clinical Oncology (ASCO) annual meeting 2019. Toripalimab, a humanized IgG4 anti-PD1 antibody was used in a phase II open label trial to treat RMNPC who had progressed on at least 2 lines of systemic treatment. One hundred and ninety patients were enrolled with no PD-L1 cut off, reporting an ORR of 25.5\% [20]. Tislelizumab was investigated in a phase I/II single arm indication-expansion study among 21 Chinese patients with RMNPC. All patients had received at least one line of systemic treatment. An ORR of $43 \%$ was observed which is the highest response rate observed in single arm studies of PD-1 inhibitors [21]. This may be due to tislelizumab being engineered to minimize binding to FcyR on macrophages in order to override antibody-dependent phagocytosis which is a potential resistance mechanism to anti PD-1 therapy. However, inter-study comparisons are fraught with limitations due to heterogeneity in patient populations and other confounding factors.

There have been two randomized phase II studies in the platinum-pretreated RMNPC setting (Table 23.2). The first among them compared the anti-PD-1 antibody spartalizumab (PDR001) monotherapy ( $\mathrm{n}=82$ patients) with chemotherapy ( $\mathrm{n}=40$ patients), the latter can be monotherapy or doublet/triplet combinations. Patients who progressed on chemotherapy were allowed to crossover to the spartalizumab arm $(n=25)$. This trial did not meet its primary PFS endpoint when spartalizumab was compared to chemotherapy (1.9 vs. 6.6 months, HR 1.36, 95\% $\mathrm{CI}=0.87-2.12)$. The ORR (95\% CI) in the spartalizumab arm versus chemotherapy 


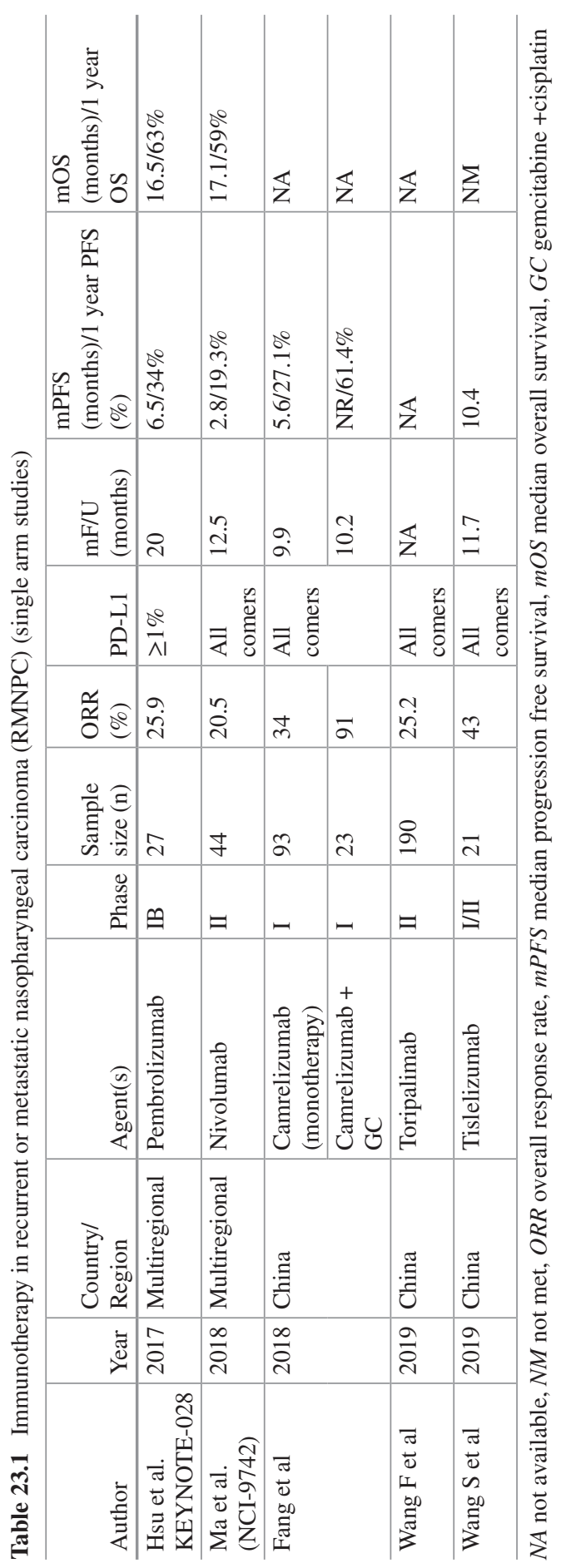




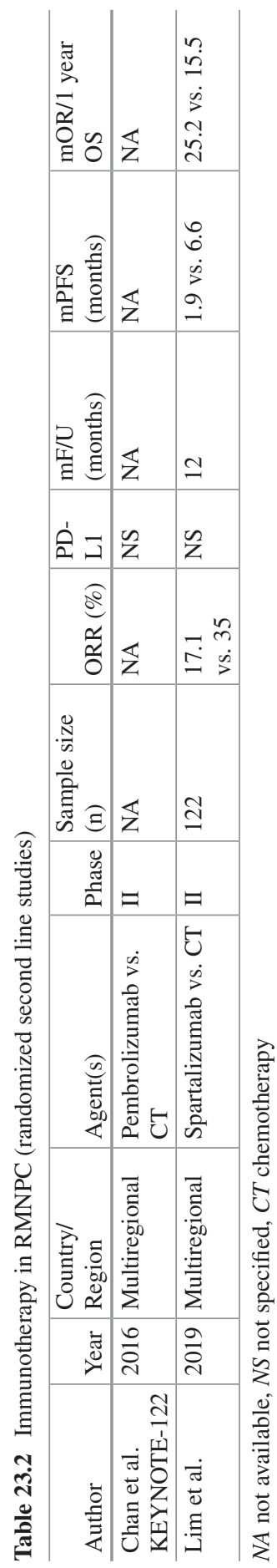


arm was $17.1 \%(9.7-27.0)$ versus $35 \%$ (20.6-51.7) respectively. The ORR for monotherapy chemotherapy was $26.9 \%$ and for doublet/triplet chemotherapy was $58.3 \%$. In the crossover arm from chemotherapy at disease progression to spartalizumab, ORR was $8.0 \%$ (1.0-26.0). However, of interest, the median duration of response was higher in the spartalizumab arm than in the chemotherapy arm (10.2 vs. 5.7 months). Median OS was also numerically longer in spartalizumab treated patients (25.2 vs. 15.5 months) but this study was not powered for this endpoint [22]. Results from the second randomized trial, KEYNOTE-122 (NCT02611960), are yet to be published.

The high response rate observed in camrelizumab in combination with chemotherapy suggests that combination of immunotherapy with chemotherapy might be the best way to elucidate response in RMNPC. Three randomized phase III trials investigating first-line anti-PD1 antibody with GC combinations are currently recruiting RMNPC patients (NCT03707509, NCT03581786, and NCT03924986) in China (Table 23.3). In addition, an international randomized study comparing nivolumab in combination with GC versus GC alone is actively being planned by the NRG cooperative group (HN007 now activated, NCT04458909).

\section{Adoptive Cell Therapy}

Adoptive cell therapy is a new therapeutic strategy based on the modulation, manipulation, and selection of autologous $\mathrm{T}$ cells in vitro to overcome the tolerance of the immune system to tumor cells. The T cells can be harvested from tumor infiltrating lymphocytes (TIL) and reinfused into the donor patient after population expansion is ensured. Lymphocyte $\mathrm{T}$ cells can also be harvested from peripheral blood, with those that recognize tumor antigens being selectively expanded. Alternatively, lymphocyte $\mathrm{T}$ cells can be genetically engineered either by modifying a $\mathrm{T}$ cell receptor for a cancer antigen ("transgenic T cell receptor" or TCR T cell) or by adding a chimeric antigen receptor that recognizes a specific cancer antigen (CAR T cell). Endemic NPC is associated with EBV, therefore targeting EBV antigens expressed

Table 23.3 Randomized First line immunotherapy combinations in RMNPC

\begin{tabular}{l|l|l|l|l|l|l}
\hline $\begin{array}{l}\text { Clinical trial } \\
\text { identifier }\end{array}$ & Investigative product & Phase & $\begin{array}{l}\text { Estimated } \\
\text { sample size }\end{array}$ & Endpoints & $\begin{array}{l}\text { Country/ } \\
\text { region }\end{array}$ & Status \\
\hline NCT03707509 & $\begin{array}{l}\text { Camrelizumab+GC } \\
\text { vs. GC }\end{array}$ & III & 250 & $\begin{array}{l}\text { PFS, ORR, } \\
\text { DCR, OS }\end{array}$ & China & Active \\
\hline NCT03581786 & $\begin{array}{l}\text { Toripalimab+GC vs. } \\
\text { GC }\end{array}$ & III & 280 & $\begin{array}{l}\text { PFS, ORR, } \\
\text { DCR, OS }\end{array}$ & $\begin{array}{l}\text { China, } \\
\text { Singapore, } \\
\text { Taiwan }\end{array}$ & Active \\
\hline NCT03924986 & $\begin{array}{l}\text { Tislelizumab+GC } \\
\text { vs. GC }\end{array}$ & III & 256 & $\begin{array}{l}\text { PFS, OS, } \\
\text { ORR, DOR }\end{array}$ & China & Active \\
\hline
\end{tabular}

$G C$ gemcitabine+cisplatin, $D C R$ duration of continued response 
in non-keratinizing and undifferentiated NPC is an attractive approach to improve outcomes for patients with advanced disease.

Adoptive transfer of EBV-specific cytotoxic T lymphocytes (EBV-CTL) as a single agent therapy has shown some benefit in phase I and II NPC studies [23-27]. Chia et al. conducted a phase II trial exploring the role of cytoreductive chemotherapy followed by autologous CTL in previously untreated patients with advanced EBV associated NPC. The patients received four cycles of carboplatin and gemcitabine followed by six doses of EBV-CTL. This combination was well tolerated and resulted in an encouraging response rate of $71.4 \%$ with 3 complete and 22 partial responses [28]. Based on these promising results, a multicenter phase III randomized controlled trial using this protocol is underway (NCT02578641). There is strong evidence of antitumor activity for EBV-CTL in patients with NPC, however response rates vary between the reported clinical trials. Contributing factors may include different technical approaches used for the generation of the EBVCTL; variable patient populations with different stages of disease, genetic predispositions, comorbidities and the impact of prior therapy. A novel approach in adoptive cell therapy is the use of allogeneic EBV-specific TIL, tabelecleucel, in combination with pembrolizumab in platinum-pretreated EBV positive RMNPC (NCT03769467). This is a multicenter, open label single-arm phase Ib/II study. Tabelecleucel will be selected for each subject from a bank of available tabelecleucel cell products based on the matching of $\geq 2$ human leucocyte antigen (HLA) alleles, at least one of which is a restricting HLA allele shared between the tabelecleucel donor and the subject's $\mathrm{EBV}+\mathrm{NPC}$.

The use of CAR T and TCR T cell therapy is relatively new in solid tumors. These represent a promising strategy that has demonstrated effective and durable responses in hematological malignancies. In a preclinical study, Tang et al. demonstrated reduced tumor growth in EBV associated NPC treated with CAR T cells [29]. Several clinical trials including phase I and II treating RMNPC with CAR T and TCR T cell therapy are ongoing (Table 23.4).

The toxic effects attributable to the activation of the host immune system have always been a major concern for adoptive cell therapy. Cytokine release syndrome (CRS) is a common toxicity observed with adoptive cell therapy. It has a heterogeneous presentation but usually involves fever, hypotension, tachycardia and respiratory insufficiency and it can be potentially fatal. The severity of CRS is correlated

Table 23.4 Ongoing CAR-T and TCR-T cell therapy trials

\begin{tabular}{l|l|l}
\hline Treatment class & Trial & Clinical trial Identifier \\
\hline CAR-T & Phase I trial of EpCAM CAR-T & NCT02915445 \\
\hline CAR-T & Phase I/II trial of LMP1-CAR-T & NCT02980315 \\
\hline CAR-T & Phase I trial of NKG2DL-CAR- $\gamma \delta-T$ & NCT04107142 \\
\hline TCR-T & Phase II trial of EBV-TCR-T (YT-E001) & NCT03648697 \\
\hline TCR-T & Phase I trial of LMP2-specific TCR-T & NCT03925896 \\
\hline
\end{tabular}

EPCAM epithelial cell adhesion molecule, CAR-T chimeric antigen receptor T cell, LMP1 latent membrane protein 1, NKG2DL natural killer group 2D ligand, $E B V$ epstein Barr virus, $L M P 2$ latent membrane protein 2 
with tumor burden [30]. Despite the toxicity profile of cell therapy approaches, the rationale of using them in EBV-directed cancer such as NPC can be justified given the presence of viral antigens that can be the target of such EBV-directed therapies.

\section{Therapeutic Vaccines}

Cancer therapeutic vaccines are designed to boost the adaptive immune response of patients by delivering different forms of tumor associated antigens into the body. Peptide based and dendritic cell (DC) vaccines have been investigated in EBV associated NPC.

A recombinant vaccinia Ankara vaccine (MVA-EL) is a peptide based vaccine that encodes inactive proteins such as full length LMP2 and C-terminal of EBNA1. This vaccine was investigated in a phase I trial in patients from Hong Kong and United Kingdom [31, 32]. The combined analysis of 27 RMNPC patients, demonstrated detectable immunologic $\mathrm{T}$ cell response to at least one vaccine coded antigen in 20 patients (74\%). A phase II study is underway for formal efficacy evaluation in RMNPC (NCT01094405).

Dendritic cells (DC) play a vital role in the activation of CD4+ and CD8+ T cells, triggering robust $\mathrm{T}$ cell immune response to the tumor antigens. Enhanced CD8+ T cell response was observed in 9 of 16 NPC patients who had been vaccinated with LMP2 peptide epitope pulsed autologous DC in a phase I trial [33]. Partial remission was observed in 2 of 16 patients. Another DC vaccine called CD137L-DCEBV-VAX is being investigated in a phase I study that is currently recruiting patients with locally advanced or RMNPC (NCT03282617).

\section{Personalized Cancer Vaccines (PCV)}

PCV are designed based on cancer specific peptides, or neoantigens, expressed by each patient's tumor tissue which harbor genomic alterations such as mutations. To create an individualized cancer vaccine, neoantigens must be identified, and then a cell-, protein- or nucleic acid based platform is used to deliver these neoantigens to patients to prime the immune system to attack the tumor. Antigen presenting cells such as DC internalize the cancer specific peptides selected for a PCV and display them on their surface with the help of major histocompatibility complex (MHC) proteins. This triggers $\mathrm{T}$ cells with receptors that bind these neoantigens to differentiate into effector, or killer T-cells that mobilize an immune reaction against cancer cells. Next generation sequencing data from tumor and normal DNA are aligned and compared to each other to identify tumor specific alterations. Neoantigens are then assessed and prioritized in order to select the ones most likely drive a robust immune response against the tumor. The selected sequences are evaluated by computer models and algorithms that predict the binding of the neoantigens to the MHC proteins 
Table 23.5 Ongoing clinical trials investigating personalized cancer vaccines (PCV)

\begin{tabular}{l|l|l|l|l|l|l}
\hline $\begin{array}{l}\text { Clinical trial } \\
\text { identifier }\end{array}$ & Phase & Cancer type & Vaccine & Other agents & $\begin{array}{l}\text { Target } \\
\text { accrual }\end{array}$ & Status \\
\hline NCT03313778 & I & $\begin{array}{l}\text { Unresectable } \\
\text { solid tumor }\end{array}$ & mRNA-4157 & Pembrolizumab & 90 & Active \\
\hline NCT03289962 & I & $\begin{array}{l}\text { Advanced solid } \\
\text { tumor }\end{array}$ & RO7198457 & Atezolizumab & 770 & Active \\
\hline NCT03662815 & I & $\begin{array}{l}\text { Advanced } \\
\text { malignant } \\
\text { tumor }\end{array}$ & $\begin{array}{l}\text { iNeo- } \\
\text { Vac-P01 }\end{array}$ & GM-CSF & 30 & Active \\
\hline NCT03568058 & I & $\begin{array}{l}\text { Advanced solid } \\
\text { tumor }\end{array}$ & $\begin{array}{l}\text { PCV (not } \\
\text { specified) }\end{array}$ & Pembrolizumab & 30 & Active \\
\hline NCT03671720 & I & $\begin{array}{l}\text { Advanced solid } \\
\text { tumor } \\
\text { (High tumor } \\
\text { mutation } \\
\text { burden) }\end{array}$ & $\begin{array}{l}\text { PCV (not } \\
\text { specified) }\end{array}$ & Cyclophosphamide & 10 & Active \\
\hline NCT02721043 & I & $\begin{array}{l}\text { Advanced solid } \\
\text { tumor }\end{array}$ & PGV001 & Poly-ICLC & 20 & Active \\
\hline
\end{tabular}

$G M$-CSF granulocyte-macrophage colony stimulating factor, $m R N A$ messenger ribonucleic acid

that would present them on the surface of cells. These PCV can be either DNA or RNA based. The promising results of early preclinical and clinical work on neoantigen vaccines have led to a number of clinical studies of personalized neoantigen vaccine based immunotherapy. Table 23.5 illustrates ongoing studies investigating PCV in solid tumors in which patients with NPC can be included.

\section{Oncolytic Viruses (OV)}

Oncolytic viruses have the ability to kill cancer cells directly as well as induce the secretion of various cytokines and chemokines to facilitate tumor antigen expression and presentation, thereby recruiting immune cells into tumors [34]. They selectively replicate in and kill cancer cells and they spread within the tumor while not harming normal tissue. They have been genetically modified to improve their safety and efficacy. OV encompass a broad diversity of DNA and RNA viruses that are naturally cancer selective. The activity of $\mathrm{OV}$ is very much a reflection of the underlying biology of the viruses from which they are derived and the host-virus interactions. Many of the hallmarks of cancer provide a permissive environment for OV; these include sustained proliferation, resisting cell death, evading growth suppressors, genome instability, DNA damage stress and avoiding immune destruction. In addition, insertion of foreign sequences can endow further selectivity for cancer cells and safety. G47 $\Delta$, a third generation herpes simplex virus 1 demonstrated some antitumor effect in EBV associated NPC [35]. There are over 10 different oncolytic viruses that have been used in antitumor research [34]. 


\section{Molecularly Targeted Agents}

Several molecularly targeted agents have been considered as second line systemic agents for RMNPC patients with a good performance status who become refractory to platinum-based regimens. Vascular endothelial growth factor receptor inhibitors and epithelial growth factor receptor inhibitors have particularly been studied in RMNPC.

\section{Vascular Endothelial Growth Factor Receptor (VEGFR) Inhibitors}

The VEGF-VEGFR interaction activates a signaling cascade that promotes angiogenesis, tumor growth and metastasis $[36,37]$. It has been shown that NPC is characterized by high expression of VEGFR-2, which in turn is adversely correlated with poor survival [38]. This mechanism has driven the development of therapies geared towards molecular targeting of VEGF-VEGFR in the management of patients with RMNPC. Axitinib, sorafenib, pazopanib, famitinib and sunitinib are multi-targeted tyrosine kinase inhibitors (TKI) of VEGFR that have demonstrated promising clinical activity in RMNPC. The efficacy of this class of agents has been demonstrated in several clinical trials of single agent VEGFR inhibitors or in combination with chemotherapy. In a comprehensive literature review by Almobarak et al. [39], the highest observed ORR of $77.8 \%$ with a median OS of 11.8 months was reported in a trial combining sorafenib with 5FU and cisplatin [40]. Axitinib demonstrated the highest ORR of $30.4 \%$ with median OS of 10.4 months as a single agent [41]. However, given the mechanisms of action, bleeding especially tumorassociated hemorrhage is a relevant concern with VEGFR inhibitors in RMNPC, especially in cases with prior high dose radiotherapy to the head and neck and in those with direct vascular invasion by tumor [42].

Newer agents such as apatinib and anlotinib have recently been explored in RMNPC. Results from a phase II trial presented at ASCO 2019 by Jiang et al. [43] demonstrated clinical activity with apatinib. Apatinib, a novel small molecule highly selective inhibitor of VEGFR-2 was given at the dose of $500 \mathrm{mg}$ daily to 33 patients across three centres in China after failure of first-line chemotherapy. This trial yielded an ORR of $36.3 \%$, with disease control rate (DCR) of 54.5\% after a median follow up time of 14 months. Median PFS of 5.0 months was observed with 1-year OS rate of $83.1 \%$. In a first-line phase III clinical trial in progress presented by Yang et al. [44] at ASCO 2019, anlotinib which is a novel multitarget TKI that targets VEGFR 1-3, fibroblast growth factor receptor 1-4 and platelet derived growth factor receptor $\alpha$ and $\beta$ was given in combination with GC compared with placebo plus GC. Fifty eight patients had been recruited out of 336 as at the time of poster presentation in ASCO. Results of this trial should be available sometime in 2021 (NCT03601975). 


\section{Epidermal Growth Factor Receptor (EGFR) Inhibitors}

The EGF-EGFR interaction activates the Ras-Raf-MEK-ERK signaling pathway, which plays various important biological roles, such as apoptosis, cell growth, cellular differentiation and malignant transformation. Non-keratinizing NPC is characterized by high expression of EGFR, as well as EGFR gene amplification in pre-clinical NPC models and patients' tumor samples. EGFR expression in NPC is associated with poor clinical and survival outcomes. Thus, molecular targeting of EGFR is a plausible therapeutic aim in recurrent and metastatic NPC. Cetuximab, gefitinib and erlotinib are EGFR inhibitors that have been studied in RMNPC [39]. Unfortunately, they have not been shown to offer meaningful clinical and survival benefits to patients with RMNPC [39].

Overall, the role of molecularly targeted agents in RMNPC has fallen short of expectations due to shortcomings that include absence of validated predictive biomarkers, small study sample sizes, lack of phase III trials and short duration of follow-up of the studies reporting molecular targeted therapy in patients with recurrent and metastatic NPC. Thus far, molecular targeted therapy in RMNPC has not been able to identify and interrogate the most important and actionable drivers in this malignancy. Furthermore, the absence of evaluation of quality of life before and after administration of molecular targeted therapy is an additional shortcoming. All these caveats, collectively, contribute to a limitation in drawing concrete conclusions. Thus, as it stands now, the role of molecular targeted therapy in patients with RMNPC remains to be further investigated.

\section{Epigenetic Therapy in RMNPC}

NPC is associated with genetic alterations on particular chromosomal regions and genes, harboring of specific cancer-associating single nucleotide polymorphisms (SNP), and familial aggregation. Interestingly, recent studies confirm that epigenetic alterations, including the promoter hypermethylation, are also one of the crucial factors that are highly associated with NPC [45].

Two genome-wide methylome studies consistently identified a few important signaling pathways and functions often deregulated by DNA methylation in NPC, including the Wnt, MAPK, Hedgehog, and TGF- $\beta$ signalling pathways and focal adhesion $[45,46]$. In the Wnt signalling pathway, a number of Wnt inhibitors including DKK1, WIF1, SFRP1, SFRP2, SFRP4, and SFRP5 are silenced by promoter methylation in NPC. Inactivation of these inhibitors may lead to the aberrant activation of Wnt signaling and transcription of its downstream targets. The enrichment of the genes with homeobox domain in the aberrantly methylated genes in NPC indicates these aberrantly methylated genes are often polycomb complex targets. Many studies have demonstrated that the polycomb repressive complex genes encoding epigenetic gene-silencing proteins contribute to the oncogenesis of various cancers. 
Overexpression of the polycomb complex genes BIMI and EZH2 was demonstrated in NPC tumor $[47,48]$.

MAK683 is an inhibitor of embryonic ectoderm development protein (EED) and allosteric inhibitor of polycomb repressive complex 2 (PRC2) with potential antineoplastic activity. There is a phase I/II clinical trial of MAK683 currently recruiting where patients with RMNPC are eligible (NCT02900651).

Notably, co-deletion of the gene encoding the methionine salvage pathway enzyme methylthioadenosine phosphorylase (MTAP), which is adjacent to the CDKN2A/p16 locus on 9p21.3 is common in NPC [49-51]. Recent studies have uncovered the pharmacologic vulnerability of MTAP-deficient tumors through drugging of the MAT2A/PRMT5/RIOK1 axis [49-51]. In MTAP/CDKN2A-deleted tumors, loss of MTAP leads to disordered methionine metabolism with impaired cleavage and accumulation of metabolite methylthioadenosine, thereby inhibiting protein arginine N-methyltransferase 5 (PRMT5) activity. Knockdown of PRMT5 and its downstream enzyme MAT2A by siRNA has shown selective growth inhibition in MTAP-deleted tumor cells [49-51]. This high frequency of MTAP loss in NPC makes PRMT5 a relevant target. There are several PRMT5 inhibitors currently being tested in clinical trials in which patients with RMNPC are a subset (Table 23.6).

Precision medicine approach on an array of druggable targets in NPC can be helpful for related subsets of patients bearing specific genomic aberrations. Once supported by more preclinical data of gene-based precision therapies for NPC, basket trials would be helpful to determine the clinical benefits of precision therapies for NPC.

\section{Conclusion and Future Approaches}

Systemic therapy in NPC represents an unmet clinical need in locally advanced and RMNPC. NPC is a complex disease that involves host genetics, viral infection, and environmental factors. It is characterized by a comparatively low mutation rate, extensive hypermethylation, as well as frequent chromosomal abnormalities and copy number alterations. Overall, EBV plays a critical role in driving the development of NPC, but it may also provide distinctive targets and opportunities for immune therapies, which warrants integrated approaches from basic science and

Table 23.6 Ongoing clinical trials investigating PRMT5

\begin{tabular}{l|l|l|l|l|l}
\hline $\begin{array}{l}\text { Clinical trial } \\
\text { identifier }\end{array}$ & Phase & Cancer type & Agents & $\begin{array}{l}\text { Target } \\
\text { accrual }\end{array}$ & Status \\
\hline NCT03573310 & I & $\begin{array}{l}\text { Advanced solid } \\
\text { tumor }\end{array}$ & JNJ-64619178 & 120 & Active \\
\hline NCT02783300 & I & $\begin{array}{l}\text { Advanced solid } \\
\text { tumor }\end{array}$ & $\begin{array}{l}\text { GSK3326595, } \\
\text { pembrolizumab }\end{array}$ & 416 & Active \\
\hline NCT04089449 & I & $\begin{array}{l}\text { Advanced } \\
\text { malignant tumor }\end{array}$ & PRT811 & 125 & Active \\
\hline
\end{tabular}


clinical perspectives. In addition, with the rapid development of precision medicine, we can optimize the combination of immunotherapy according to the different genomic characteristics and immune status of individual patients and customize therapy to obtain the maximum clinical effect.

\section{Financial Disclosures}

Olubukola Ayodele:

- None.

Lillian L. Siu:

- Stock ownership or equity: Agios (spouse).

- Employee, office, directorship: none.

- Leadership in: Treadwell therapeutics (spouse = co-founder).

- Consulting/advisory arrangements: Merck (compensated), Pfizer (compensated), Celgene (compensated), AstraZeneca/Medimmune (compensated), Morphosys (compensated), Roche (compensated), GeneSeeq (compensated), Loxo (compensated), Oncorus (compensated), Symphogen (compensated), Seattle Genetics (compensated), GSK (compensated), Voronoi (compensated), Treadwell Therapeutics (compensated), Arvinas (compensated), Tessa (compensated), Navire (compensated), Relay (compensated), Rubius (compensated).

- Speaker's Bureau for: none.

- Grant/Research support (Clinical Trials for institution): Novartis, Bristol-Myers Squibb, Pfizer, Boerhinger-Ingelheim, GlaxoSmithKline, Roche/Genentech, Karyopharm, AstraZeneca/ Medimmune, Merck, Celgene, Astellas, Bayer, Abbvie, Amgen, Symphogen, Intensity Therapeutics, Mirati, Shattucks, Avid.

- Travel grants: none.

- Intellectual property rights: none.

\section{References}

1. Lee AW, Poon YF, Foo W, et al. Retrospective analysis of 5037 patients with nasopharyngeal carcinoma treated during 1976-1985: overall survival and patterns of failure. Int J Radiat Oncol Biol Phys. 1992;23:261-70.

2. Li AC, Xiao WW, Shen GZ, et al. Distant metastasis risk and patterns of nasopharyngeal carcinoma in the era of IMRT: long-term results and benefits of chemotherapy. Oncotarget. 2015;6:24511-21.

3. Tang LQ, Chen QY, Fan W, et al. Prospective study of tailoring whole-body dual-modality [18F] fluorodeoxyglucose positron emission tomography/computed tomography with plasma Epstein-Barr virus DNA for detecting distant metastasis in endemic nasopharyngeal carcinoma at initial staging. J Clin Oncol. 2013;31:2861-9.

4. Chen FH, Wang ZQ, An X, et al. Salvage gemcitabine-vinorelbine chemotherapy in patients with metastatic nasopharyngeal carcinoma pretreated with platinum-based chemotherapy. Oral Oncol. 2012;48:1146-51.

5. Leong SS, Wee J, Rajan S, et al. Triplet combination of gemcitabine, paclitaxel, and carboplatin followed by maintenance 5-fluorouracil and folinic acid in patients with metastatic nasopharyngeal carcinoma. Cancer. 2008;113:1332-7. 
6. Zhang L, Huang Y, Hong S, et al. Gemcitabine plus cisplatin versus fluorouracil plus cisplatin in recurrent or metastatic nasopharyngeal carcinoma: a multicentre, randomised, open-label, phase 3 trial. Lancet. 2016;388:1883-1.

7. Chang ET, Adami HO. The enigmatic epidemiology of nasopharyngeal carcinoma. Cancer Epidemiol Biomark Prev. 2006;15:1765-77.

8. Tsao SW, Yip YL, Tsang CM, et al. Etiological factors of nasopharyngeal carcinoma. Oral Oncol. 2014;50:330-8.

9. Raghupathy R, Hui EP, Chan AT. Epstein-Barr virus as a paradigm in nasopharyngeal cancer: from lab to clinic. Am Soc Clin Oncol Educ Book. 2014:149-53.

10. Fang W, Zhang J, Hong S, et al. EBV-driven LMP1 and IFN-g up-regulate PD-L1 in nasopharyngeal carcinoma: implications for oncotargeted therapy. Oncotarget. 2014;5:12189-202.

11. Zhang J, Fang W, Qin T, et al. Coexpression of PD-1 and PD-L1 predicts poor outcome in nasopharyngeal carcinoma. Med Oncol. 2015;32:86.

12. Chen BJ, Chapuy B, Ouyang J, et al. PD-L1 expression is characteristic of a subset of aggressive B-cell lymphomas and virus-associated malignancies. Clin Cancer Res. 2013;19:3462-73.

13. Lu J, Chen XM, Huang HR, et al. Detailed analysis of inflammatory cell infiltration and the prognostic impact on nasopharyngeal carcinoma. Head Neck. 2018;40(6):1245-53.

14. Huang SCM, Tsao SW, Tsang CM. Interplay of viral infection, host cell factors and tumor microenvironment in the pathogenesis of nasopharyngeal carcinoma. Cancers (Basel). 2018;10(4):106.

15. Zhang YL, Li J, Mo HY, et al. Different subsets of tumor infiltrating lymphocytes correlate with NPC progression in different ways. Mol Cancer. 2010;10(9):4.

16. Hanahan D, Weinberg RA. Hallmarks of cancer: the next generation. Cell. 2011;144:646-74.

17. Hsu C, Lee SH, Ejadi S, et al. Safety and antitumor activity of pembrolizumab in patients with programmed Death-Ligand 1-positive nasopharyngeal carcinoma: results of the KEYNOTE-028 study. J Clin Oncol. 2017;35(36):4050-6.

18. Ma BBY, Lim WT, Goh BC, et al. Antitumor activity of nivolumab in recurrent and metastatic nasopharyngeal carcinoma: an international, multicenter study of the Mayo Clinic phase 2 Consortium (NCI-9742). J Clin Oncol. 2018;36(14):1412-8.

19. Fang W, Yang Y, Ma Y, et al. Camrelizumab (SHR-1210) alone or in combination with gemcitabine plus cisplatin for nasopharyngeal carcinoma: results from two single-arm, phase 1 trials. Lancet Oncol. 2018;19(10):1338-50.

20. Wang F, Wei XL, Feng JF, et al. Recombinant humanized anti-PD-1 monoclonal antibody (JS001) in patients with refractory/metastatic nasopharyngeal carcinoma: interim results of an open-label phase II clinical study. J Clin Oncol. 2019;37(Suppl 15):6017.

21. Wang S, Huang X, Bai YX, et al. Preliminary results with tislelizumab, an investigational anti-PD-1 antibody, in Chinese patients with nasopharyngeal cancer (NPC). J Clin Oncol. 2019;37(Suppl 15):2556.

22. Lim DW, Wang HM, Li SH, et al. Abstract CT150: phase II study of spartalizumab (PDR001) vs chemotherapy (CT) in patients with recurrent/metastatic nasopharyngeal cancer (NPC). Cancer Res. 2019;79(Suppl 13):CT150.

23. Chua D, Huang J, Zheng B, et al. Adoptive transfer of autologous Epstein-Barr virus-specific cytotoxic T cells for nasopharyngeal carcinoma. Int J Cancer. 2001;94:73-80.

24. Comoli P, De Palma R, Siena S, et al. Adoptive transfer of allogeneic Epstein-Barr virus (EBV)-specific cytotoxic T cells with in vitro antitumor activity boosts LMP2-specific immune response in a patient with EBV-related nasopharyngeal carcinoma. Ann Oncol. 2004;15:113-7.

25. Comoli P, Pedrazzoli P, Maccario R, et al. Cell therapy of stage IV nasopharyngeal carcinoma with autologous Epstein-Barr virus-targeted cytotoxic T lymphocytes. J Clin Oncol. 2005;23:8942-9.

26. Straathof KC, Bollard CM, Popat U, et al. Treatment of nasopharyngeal carcinoma with Epstein-Barr virus-specific T lymphocytes. Blood. 2005;105:1898-904.

27. Louis CU, Straathof K, Bollard CM, et al. Adoptive transfer of EBV-specific T cells results in sustained clinical responses in patients with locoregional nasopharyngeal carcinoma. $\mathrm{J}$ Immunother. 2010;33:983-90. 
28. Chia WK, Teo M, Wang WW, et al. Adoptive T-cell transfer and chemotherapy in the firstline treatment of metastatic and/or locally recurrent nasopharyngeal carcinoma. Mol Ther. 2014;22:132-9.

29. Tang X, Zhou Y, Li W, et al. T-cells expressing a LMP1-specific chimeric antigen receptor mediate antitumor effects against LMP1-positive nasopharyngeal carcinoma cells in vitro and in vivo. J Biomed Res. 2014;28(6):468-75.

30. Hay KA, Hanafi L-A, Li D, et al. Kinetics and biomarkers of severe cytokine release syndrome after CD19 chimeric antigen receptor-modified T-cell therapy. Blood. 2017;130(21):2295-306. https://doi.org/10.1182/blood-2017-06-793141.

31. Hui EP, Taylor GS, Jia H, et al. Phase I trial of recombinant modified vaccinia ankara encoding Epstein-Barr viral tumor antigens in nasopharyngeal carcinoma patients. Cancer Res. 2013;73(6):1676-88.

32. Taylor GS, Jia H, Harrington $\mathrm{K}$, et al. A recombinant modified vaccinia ankara vaccine encoding Epstein-Barr Virus (EBV) target antigens: a phase I trial in UK patients with EBV-positive cancer. Clin Cancer Res. 2014;20(19):5009-22.

33. Lin CL, Lo WF, Lee TH, et al. Immunization with Epstein-Barr virus (EBV) peptide-pulsed dendritic cells induces functional CD8+ T-cell immunity and may lead to tumor regression in patients with EBV-positive nasopharyngeal carcinoma. Cancer Res. 2002;62(23):6952-8.

34. Kaufman HL, Kohlhapp FJ, Zloza A. Oncolytic viruses: a new class of immunotherapy drugs. Nat Rev Drug Discov. 2015;14(9):642-62.

35. Wang JN, Hu P, Zeng MS, Liu RB. Anti-tumor effect of oncolytic herpes simplex virus G47delta on human nasopharyngeal carcinoma. Chinese J Cancer. 2011;30(12):831-41.

36. Ferrara N, Kerbel RS. Angiogenesis as a therapeutic target. Nature. 2005;438:967-74.

37. Ferrara N, Adamis AP. Ten years of anti-vascular endothelial growth factor therapy. Nat Rev Drug Discov. 2016;15:385-403.

38. Hui EP, Chan AT, Pezzella F, et al. Coexpression of hypoxia-inducible factors 1alpha and 2alpha, carbonic anhydrase IX, and vascular endothelial growth factor in nasopharyngeal carcinoma and relationship to survival. Clin Cancer Res. 2002;8:2595-604.

39. Almobarak AA, Jebreel AB, Abu-Zaid A. A molecular targeted therapy in the management of recurrent and metastatic nasopharyngeal carcinoma: a comprehensive literative review. Cureus. 2019;11(3):e4210.

40. Xue C, Huang Y, Huang PY, et al. Phase II study of sorafenib in combination with cisplatin and 5-fluorouracil to treat recurrent or metastatic nasopharyngeal carcinoma. Ann Oncol. 2013;24:1055-61.

41. Hui EP, Ma BBY, Loong HHF, et al. Efficacy, safety, and pharmacokinetics of axitinib in nasopharyngeal carcinoma: a preclinical and phase II correlative study. Clin Cancer Res. 2018;24:1030-7.

42. Hui EP, Ma BB, King AD, et al. Hemorrhagic complications in a phase II study of sunitinib in patients of nasopharyngeal carcinoma who has previously received high-dose radiation. Ann Oncol. 2011;22(6):1280-7. https://doi.org/10.1093/annonc/mdq629.

43. Jiang W, Liang J, Pan Y, et al. Apatinib for locoregionally recurrent or metastatic nasopharyngeal carcinoma after failure of first-line chemotherapy: a multicenter, phase II trial. J Clin Oncol. 2019;37(Suppl 15):6030.

44. Yang Y, Huang Y, Fang W, et al. A multicenter, randomized, double-blind, placebo-controlled phase III study of anlotinib or placebo in combination with gemcitabine and cisplatin (GP) as first-line treatment for recurrent or metastatic nasopharyngeal carcinoma (R/M NPC). J Clin Oncol. 2019;37(Suppl 15):TPS6089.

45. Dai W, Cheung AK, Ko JM, et al. Comparative methylome analysis in solid tumors reveals aberrant methylation at chromosome $6 \mathrm{p}$ in nasopharyngeal carcinoma. Cancer Med. 2015;4:1079-90.

46. Li L, Zhang Y, Fan Y, et al. Characterization of the nasopharyngeal carcinoma methylome identifies aberrant disruption of key signaling pathways and methylated tumor suppressor genes. Epigenomics. 2015;7:155-73. 
47. Alajez NM, Shi W, Hui AB, et al. Enhancer of Zeste homolog 2 (EZH2) is overexpressed in recurrent nasopharyngeal carcinoma and is regulated by miR-26a, miR-101, and miR-98. Cell Death Dis. 2010;1:e85.

48. Song LB, Zeng MS, Liao WT, et al. Bmi-1 is a novel molecular marker of nasopharyngeal carcinoma progression and immortalizes primary human nasopharyngeal epithelial cells. Cancer Res. 2006;66:6225-32.

49. Kryukov GV, Wilson FH, Ruth JR, Paulk J, Tsherniak A, Marlow SE, et al. MTAP deletion confers enhanced dependency on the PRMT5 arginine methyltransferase in cancer cells. Science. 2016;351(6278):1214-8.

50. Marjon K, Cameron MJ, Quang P, Clasquin MF, Mandley E, Kunii K, et al. MTAP deletions in cancer create vulnerability to targeting of the MAT2A/PRMT5/RIOK1 Axis. Cell Rep. 2016;15(3):574-87.

51. Mavrakis KJ, McDonald ER 3rd, Schlabach MR, Billy E, Hoffman GR, de Weck A, et al. Disordered methionine metabolism in MTAP/CDKN2A-deleted cancers leads to dependence on PRMT5. Science. 2016;351(6278):1208-13.

Open Access This chapter is licensed under the terms of the Creative Commons Attribution 4.0 International License (http://creativecommons.org/licenses/by/4.0/), which permits use, sharing, adaptation, distribution and reproduction in any medium or format, as long as you give appropriate credit to the original author(s) and the source, provide a link to the Creative Commons license and indicate if changes were made.

The images or other third party material in this chapter are included in the chapter's Creative Commons license, unless indicated otherwise in a credit line to the material. If material is not included in the chapter's Creative Commons license and your intended use is not permitted by statutory regulation or exceeds the permitted use, you will need to obtain permission directly from the copyright holder.

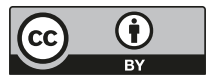

